\title{
Nanoparticulate delivery of novel drug combination regimens for the chemoprevention of colon cancer
}

\author{
NAVEEN KANTHAMNENI ${ }^{1}$, ABHISHEK CHAUDHARY ${ }^{2}$, JEFFREY WANG $^{2}$ and SUNIL PRABHU ${ }^{2}$ \\ ${ }^{1}$ College of Pharmacy, The Ohio State University, 500W 12th Avenue, Columbus, OH 43210; \\ ${ }^{2}$ Department of Pharmaceutical Sciences, College of Pharmacy, Western University \\ of Health Sciences, 309 East 2nd Street, Pomona, CA 91766, USA
}

Received February 16, 2010; Accepted March 15, 2010

DOI: 10.3892/ijo_00000665

\begin{abstract}
The purpose of this work was to assess synergistic inhibitory responses of a novel chemopreventive combination regimen of drugs namely, aspirin in combination with calcium and folic acid on two human colon cancer cell lines, HT-29 and SW-480. Subsequently, based on positive responses, nanotechnology-based formulations were developed for the targeted delivery of these combinatorial regimens to the colon for the chemoprevention of colon cancer. Additionally, conventional drug formulations using controlled release polymers chitosan, pectin and hydroxypropyl methylcellulose (HPMC) were tested for release of the drugs, for comparison purposes. Chemopreventive combination regimens demonstrated significant synergistic efficacy in both cell lines from XTT assay studies, when compared to the effects of individual agents. Approximately $45 \%$ decrease in cell viability for aspirin $(15 \mathrm{mM})$ and calcium $(30 \mathrm{mM})$ mixtures was observed in HT-29 cell lines, compared to 55\% decrease by the same combination in SW-480 cell lines. With combinations of aspirin $(5 \mathrm{mM})$ and folic acid (1.5 mM), HT-29 cells demonstrated a $30 \%$ decrease in cell viability compared to $\sim 38 \%$ decrease in the SW-480 cell line. Overall, all drug combinations demonstrated significant synergistic responses in the cell lines tested with the SW-480 cell line being more significantly affected by the drug regimens than the HT-29 cell line. Drug encapsulated nanoparticles demonstrated a spherical morphology, $<125 \mathrm{~nm}$ average particle size (aspirin and folic acid) of nanoparticles and encapsulation efficiencies in the range of $80-91 \%$. Drug release from nanoparticles was controlled with $\sim 60 \%$ of the original amount released over a $96 \mathrm{~h}$ period. Conventional formulations exhibited faster kinetics of drug release when compared to the PLGA nano-
\end{abstract}

Correspondence to: Dr Sunil Prabhu, Department of Pharmaceutical Sciences, College of Pharmacy, Western University of Health Sciences, 309 East 2nd Street, Pomona, CA 91766, USA E-mail: sprabhu@westernu.edu

Key words: chemoprevention, colon cancer, combination therapy, nanotechnology particles. Overall, the cell line studies demonstrate, for the first time, the ability of novel chemopreventive combinations to inhibit the growth of colon cancer cells whereas the nanotechnology-based drug delivery system provides valuable evidence for targeted therapy towards colon cancer chemoprevention.

\section{Introduction}

According to the American Cancer Society (ACS), colorectal (CRC) cancer is the third most common cancer in both men and women. An estimated 106,100 cases of colon and 40,870 cases of colorectal cancer were expected to occur in 2009 (ACS Cancer Statistics 2009) from which an estimated 49,920 deaths would result accounting for almost $9 \%$ of all cancer deaths. Cancer of the colon arises due to a series of histopathological and molecular changes that result in the over-expression of epithelial cells transforming into a colorectal carcinoma, with adenomatous polyps and aberrant cryptic foci (ACF) as early intermediates in the process (1).

Current research is shifting the focus of CRC disease from diagnosis and treatment to chemoprevention (2). Chemoprevention aims to prevent the development or recurrence of precancerous lesions and cancers with the use of natural or synthetic agents that reverse, suppress, delay, or prevent carcinogenic progression to invasive disease (3). The development of cancer results not only from a single genetic event but from accumulation of a number of molecular genetic alterations. By interfering with these molecular events, chemoprevention could inhibit or reverse the development of adenomas or the progression from adenoma to cancer.

Some of the best studied chemopreventive agents against colon cancer include aspirin, folic acid and calcium (4). As a chemopreventive agent, aspirin-based clinical trials have demonstrated a 40-50\% decrease in colon cancer mortality in humans with a high aspirin intake (5). Studies with folic acid showed a $31 \%$ reduction in risk for colorectal adenomas and colon cancer after prolonged folate supplementation of $400 \mu \mathrm{g}$ /day (6). Among non-pharmacologic agents, calcium supplementation has shown an inverse association between calcium intake and colorectal neoplasia. Oral elemental calcium therapy, 2.4 or $3.6 \mathrm{~g} / \mathrm{day}$, for three months markedly reduced fecal DAG concentration and output without enhancing DAG production (7). 
Recent literature has shown that rather than administering single agents, there is an increasing interest in the use of combinations of chemopreventive agents that differ in mode of action. This approach provides means of obtaining lowdose therapy, increased efficacy and minimized toxicity when promising chemopreventive agents demonstrate toxic effects at higher doses (8). No group has investigated the combined therapeutic effects of aspirin and folic acid or calcium on the prevention of CRC. Since all of these agents have different mechanisms of action in chemoprevention, an anticipated synergistic effect in CRC reduction would provide valuable information in assessing combination chemopreventive regimes for eventual clinical use.

Thus, we tested a novel approach of combining the above chemopreventive agents to exert a local synergistic effect in the colonic epithelia using two colon cancer cell lines, HT-29 (Cox-2 enzyme expressive) and SW-480 (Cox-2 enzyme deficient), as surrogate biomarkers. Based on previous studies demonstrating the effect of increasing doses of aspirin on cell viability (9) chemopreventive combination doses were used which were higher than normal therapeutic levels, to test for synergistic activity of the chemopreventive combinations.

The method of delivery plays an important role in ensuring that the drug reaches its target site for therapeutic effect. Novel modes of delivery methods using microsphere and nanosphere technology $(10,11)$ are receiving wide attention as these have shown superior delivery compared to conventional dosage forms. Despite evidence of benefits of such carrier systems, its application in chemoprevention has never been studied, demonstrating a need for research in this area. Nanoparticulate drug delivery systems offer several advantages such as controlled and sustained release of drugs, ability to cross the mucosal barriers, decreased renal and hepatic clearance, decreased immune recognition, increased halflives of drugs due to encapsulation and slow release from polymers, enhanced intracellular uptake thereby allowing drug release in different cellular compartments, increased stability and solubility of drugs as well as reduce drug resistance in some human carcinomas (12). Additionally, nanoparticles have a large specific surface which is indicative of a high interactive potential with biological surfaces. Such multi-particulate systems tend to be more uniformly dispersed in the GI tract and ensure uniform absorption (13).

Thus, using an FDA approved, biodegradable co-polymer, poly-(lactide-co-glycolide) (PLGA) for the preparation of drug-loaded nanoparticles (14), combinations of chemopreventive agents were encapsulated within nanoparticles and targeted directly to the colon for the synergistic chemoprevention of colon cancer. Targeting was achieved by coating known mixtures of aspirin and folic acid encapsulated polymer nanoparticles with a commercially available colonic polymer Eudragit ${ }^{\circledR}$ S-100. For this study, calcium was not encapsulated. Eudragit S-100 class of polymer dissolves at the colonic $\mathrm{pH}$ but resists lower $\mathrm{pH}$ activity in the stomach or the small intestine (15). No other group has investigated this novel approach for the chemoprevention of colon cancer to date although studies have been reported for treatment of inflammatory bowel disease (I.B.D.) using drug-loaded PLGA nanoparticles targeted to the colon (16). Additional studies were conducted to compare the in vitro release characteristics of chemopreventive agents encapsulated within conventional colonic polymers such as chitosan, pectin and hydroxypropyl methylcellulose (HPMC) to that of drug loaded PLGA nanoparticles.

\section{Materials and methods}

Materials. The biodegradable polymers, low molecular weight chitosan, pectin and hydroxypropyl methycellulose (HPMC) polymers were purchased from Sigma-Aldrich (St. Louis, MO) as were aspirin, folic acid and calcium. Eudragit S100, an enteric-coated colonic polymer used for coating the capsules was gifted by Degussa Corp. (Parsippany, NJ). Polylactide-co-glycolide (PLGA) copolymers were purchased from Durect Corp. (Pelham, AL).

Human cell lines. Human colon adenocarcinoma cells, HT-29 (Cox-2 enzyme expressive) and SW-480 (Cox-2 enzyme deficient) were purchased from American Type Culture Collection, ATCC (Manassas, VA). The media required for the growth of cells, Leibovitz L15 media for SW-480, McCoy's media for the growth of HT-29 cell lines and fetal bovine serum (FBS) were purchased from Hyclone Laboratories (Manassas, VA). Cells were cultured at $37^{\circ} \mathrm{C}$ in a humidified atmosphere of $5 \% \mathrm{CO}_{2}$ and $95 \%$ air.

Cell viability (XTT) assay. HT-29 cells were grown in McCoys medium supplemented with $10 \%$ fetal bovine serum in an incubator at $37^{\circ} \mathrm{C}$ under $5 \% \mathrm{CO}_{2}$ atmosphere. SW-480 was developed in an incubator with $100 \%$ air, using Leibovitz's L-15 medium with $2 \mathrm{mM}$ L-glutamine, $10 \%$ fetal bovine serum. The cell viability following treatment with chemopreventive combinations of aspirin with calcium or folic acid was determined by using XTT colorimetric assay at $450 \mathrm{~nm}$. SW-480 or HT-29 cells were seeded in 96-well plates at a density of $5 \times 10^{3}$ cells/well, in $200 \mu 1$ of growth medium and incubated for $12 \mathrm{~h}$ at $37^{\circ} \mathrm{C}$ under $5 \% \mathrm{CO}_{2}$ atmosphere. After $12 \mathrm{~h}$, the cells were treated with the chemopreventive combinations for another 24-96 h. Each vial of XTT to be used was reconstituted with $5 \mathrm{ml}$ of the medium without phenol red and serum. Finally, the cells were incubated with XTT containing medium $(0.5 \mathrm{mg} / \mathrm{ml}$ XTT in medium) for $2-6 \mathrm{~h}$, depending on the cell type. Gentle mixing was done using a vortex machine to enhance dispersion. The absorbance was measured spectrophotometrically at $450 \mathrm{~nm}$ using a microplate reader (Bio-Tek, VT, USA). The studies were conducted for the duration of $96 \mathrm{~h}$ hence four sets of 96-well plates were prepared for each formulation.

Preparation of novel controlled release formulations. The nanotechnology-based formulations of aspirin and folic acid were prepared using a water/oil/water (w/o/w) emulsion process of solvent evaporation (17). Aspirin and folic acid were dissolved or suspended in an aqueous medium, and emulsified in organic solution of dichloromethane containing PLGA copolymers. For the w/o/w emulsion using aspirin, PLGA 50:50 was dissolved in dichloromethane (DCM) (oil phase) and emulsified with aspirin in $1 \%$ polyvinyl alcohol (PVA, used as stabilizer) solution (water phase). The emulsion 
was probe sonicated (Branson, Los Angeles, CA) for $2 \mathrm{~min}$ initially and added dropwise to an outer aqueous $2 \%$ PVA solution to complete the formation of the multiple emulsion. The DCM solvent was evaporated resulting in the precipitation of drug-loaded nanoparticles. To recover the nanoparticles, each formulation was subjected to ultracentrifugation at $10^{\circ} \mathrm{C}$ for $25 \mathrm{~min}$ at 34,500 x g (Beckman L8-80M, Los Angeles, CA). The supernatant was collected, and the resulting nanoparticle sediment was washed again with water to remove excess drug and PVA from the surface of the nanoparticles. Finally, the nanoparticles were freeze-dried (Labconco, Kansas City, MO) and stored at $4^{\circ} \mathrm{C}$ until further use. Similarly, folic acid formulations were prepared with different grades of PLGA using the w/o/w emulsion methodology. For targeting to the colon, mixtures of freeze-dried aspirin and folic acid-encapsulated polymer nanoparticles were coated with a commercially available colonic polymer Eudragit S-100 dissolved in methanol $(1 \% \mathrm{w} / \mathrm{v})$. The treated nanoparticles were allowed to dry for $1 \mathrm{~h}$ before sieving the powder sample through a sieve no. 30 for even sized particles.

Physical mixtures of aspirin and folic acid were prepared using combinations of chitosan, pectin or HPMC in a 1:2 proportion. Known weights of the mixtures were filled into capsules (size 00) using a capsule machine (Janson, Napa, CA). Samples were prepared in triplicate.

Particle sizing and morphology. The size and size distribution of the prepared PLGA nanoparticles were measured by using light scattering size analyzer (Nicomp sub-micron analyzer, Model 370, Santa Barbara, CA). The analysis was performed at a scattering angle of $90^{\circ}$ and at a temperature of $25^{\circ} \mathrm{C}$. Upon evaporation of the organic solvent, each of the nanoparticle-based dispersions was subjected to size analysis. A $1 \mathrm{ml}$ sample of the dispersion was diluted with water (X10). The diluted samples were placed in cuvettes and analyzed. The obtained homogeneous suspension was assessed for mean diameter (nm) and size distribution (\%) in triplicate.

Nanoparticle morphology was evaluated using atomic force microscopy (AFM) (Agilent, Santa Clara, CA) whereby $5 \mathrm{mg}$ of freeze-dried nanoparticle sample was suspended in $10 \mathrm{ml}$ of methanol and sonified for $3 \mathrm{~min}$. The suspension was filtered through a $0.45-\mu$ filter, placed on glass microscope slides and dried in a desiccator for $24 \mathrm{~h}$ before being placed in the analysis chamber of the AFM for morphology studies.

Determination of encapsulation efficiency (\%). The amount of the drug entrapped within the nanoparticle was determined by measuring the non-entrapped (un-encapsulated) drug amount in the supernatant. The supernatant was collected after ultracentrifugation and recovery of nanoparticles. The encapsulation was analyzed by UV-Vis spectrophotometric analysis (Shimadzu, Japan) at $275 \mathrm{~nm}$ for aspirin and $282 \mathrm{~nm}$ for folic acid. Encapsulation efficiency was determined by using the following expression (18):

$$
\text { E.E. }(\%)=\frac{W_{t}-W_{u}}{W_{t}} \times 100
$$

where, Wt, weight of initial drug; Wu, weight of un-encapsulated drug.
In vitro drug release studies. For release studies, drug-encapsulated PLGA 50:50 nanoparticles (30 mg) were dispersed in a capped centrifuge tube containing $10 \mathrm{ml}$ of PBS (pH 7.4). The tube was placed in an orbital shaker water bath at $37^{\circ} \mathrm{C}$. At designated intervals, $3 \mathrm{ml}$ of the sample was withdrawn using a $0.22-\mu \mathrm{m}$ filter loaded to a syringe and then replaced with fresh buffer solution. Samples were analyzed at a wavelength of $275 \mathrm{~nm}$ for aspirin and $282 \mathrm{~nm}$ for folic acid using an UV-Vis spectrophotometer.

For conventional formulations, in vitro dissolution studies were carried out using USP type I basket apparatus model VK-7000 (Vankel, Cary, NC). The basket rotation was maintained at $50 \mathrm{rpm}$ and the temperature was set at $37 \pm 0.5^{\circ} \mathrm{C}$. The drug release studies were conducted in $900 \mathrm{ml}$ of phosphate buffer solution at a $\mathrm{pH}$ of 7.4 for $5 \mathrm{~h}$ to simulate the colonic environment. Samples were collected and analyzed by spectrophotometric analysis at the same wavelengths as stated above.

Statistical analysis. The data from the cell culture studies were analyzed by student's t-test and analysis of variance (one way). The GraphPad Prism ${ }^{\circledR}$ version 5.02 software (GraphPad Software, Inc., La Jolla, CA) was used for the statistical analysis.

\section{Results}

Cell viability studies on HT-29 and SW-480 colon adenocarcinoma cell lines. Both cell lines, HT-29 (cox-2 enzyme expressive) and SW-480 (cox-2 enzyme deficient) were incubated for $48 \mathrm{~h}$ after being treated with mixtures of aspirin $(15 \mathrm{mM})$ and calcium $(30 \mathrm{mM})$. As shown in Fig. 1a, individual concentrations of aspirin and calcium did not demonstrate any significant decrease in colon cancer cell viability although aspirin by itself showed a slight decrease ( 20\%). However, when mixed together in the same concentrations and upon incubation for $48 \mathrm{~h}$, a further decrease of $\sim 45 \%$ in cell viability was observed which indicated a synergistic response of the combined drug regimen. Calcium by itself did not show any decrease in cell viability, on the contrary showed a slight increase which suggested that it may actually be acting as a supplement causing an increase in cell growth at the concentration tested. This result was consistent with previous studies in literature that have demonstrated similar action of calcium (19). In combination with aspirin however, the decrease in cell viability was significant as shown above.

SW-480 is a cox-2 enzyme-deficient colon cancer cell line (20). Fig. 1b shows results similar to the HT-29 cell line with individual concentrations of aspirin and calcium demonstrating low to moderate changes in SW-480 cell viability. When combined together, a synergistic effect equivalent to $\sim 55 \%$ decrease in cell viability was observed $(\mathrm{p}<0.05)$. The magnitude of the effect of drug combinations on the reduction of cell viability in SW-480 cell line was more pronounced than that of the HT-29 cell line, thus demonstrating that aspirin and calcium combinations are more effective in cox-2 enzyme deficient cell lines.

The chemopreventive effects of the combined regimen were similarly tested on the two cell lines using aspirin in combination with low concentrations of folic acid. As shown 

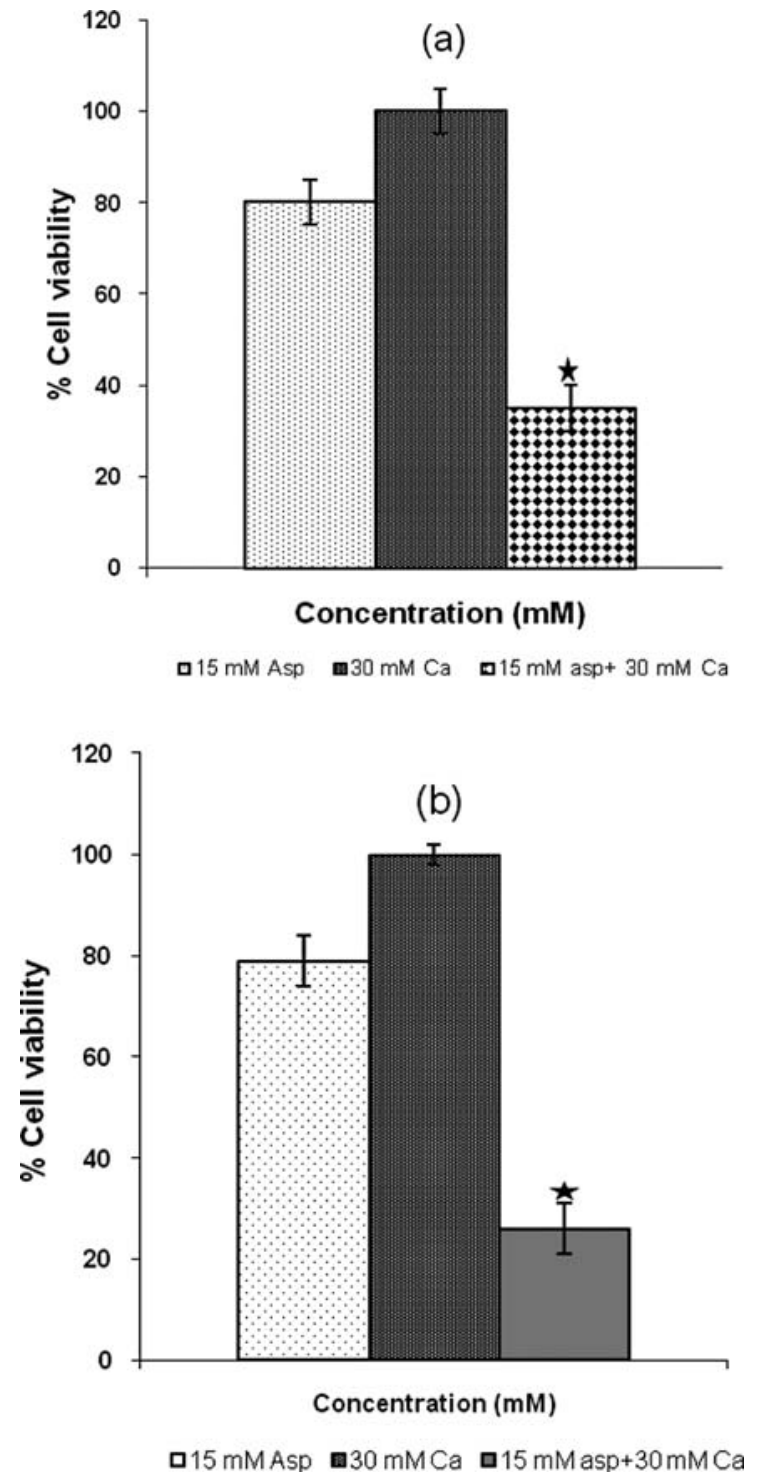

Figure 1. Synergistic effect of aspirin (Asp) and calcium (Ca) mixtures in the reduction of cell viability in (a) HT-29 and (b) SW-480 colon cancer cell lines after $48 \mathrm{~h}$ incubation and $6 \mathrm{~h}$ staining period $\left({ }^{*} \mathrm{p}<0.05\right)$.

in Fig. 2a, a combined mixture of aspirin $(5 \mathrm{mM})$ and folic acid $(1.5 \mathrm{mM})$ shows a $50 \%$ decrease in cancer cell viability in the HT-29 cell line. The synergism was also evident in the SW-480 cell line (Fig. 2b) whereby 58\% inhibition of cell viability was measured. In either cell line, the concentration of folic acid by itself did not have any effect on the reduction of cell viability but when combined with aspirin showed a significant effect. The magnitude of decrease in cell viability of aspirin combined with folic acid was smaller than when combined with calcium, however the decrease was significant $(\mathrm{p}<0.05)$ when compared to individual concentrations of the chemopreventive agents. These results were consistent with previous studies reported in literature (20).

PLGA 50:50 nanoparticle surface morphology and particle size analysis. Surface topography and 3-D images of the PLGA 50:50 copolymer nanoparticles were observed by atomic force microscopy (AFM). As shown in Fig. 3a and b, the images demonstrate a fairly rounded structure in the nano-
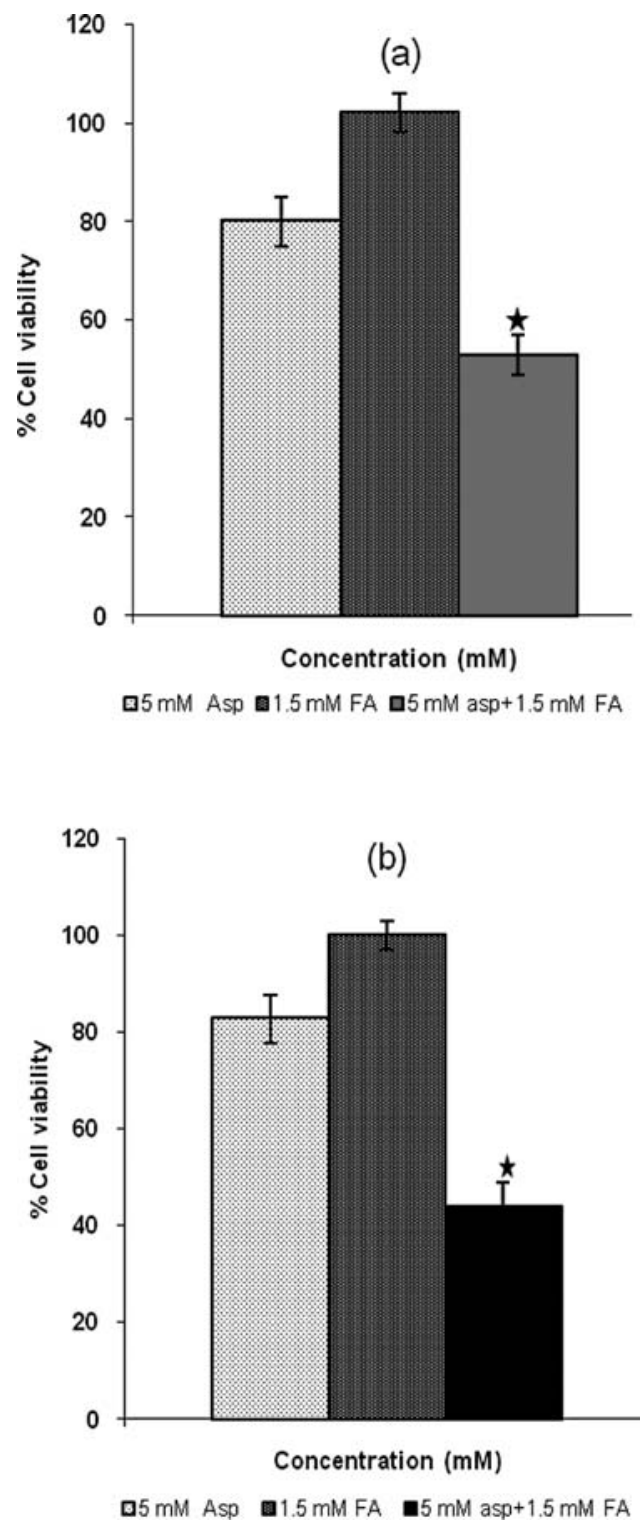

Figure 2. Synergistic effect of aspirin (Asp) and folic acid (FA) mixtures in the reduction of cell viability in (a) HT-29 and (b) SW-480 colon cancer cell lines after $48 \mathrm{~h}$ incubation and $6 \mathrm{~h}$ staining period $\left({ }^{*} \mathrm{p}<0.05\right)$.

meter range when viewed from the top (Fig. 3a). The 3-D orientation in Fig. 3b shows the nanoparticle distribution and peak heights of the PLGA 50:50 nanoparticles exhibiting sharp contours with smooth sides. Overall, these images demonstrated the surface morphology of the nanoparticles thus providing a qualitative assessment of the prepared formulation.

The determination of size, size distribution are important to be verified in the development of nanoparticles (21). Particle size analysis of nanoparticles containing aspirin in increasing concentrations $(20,40$ and $60 \%)$ in two PLGA polymers (50:50 and 85:15) is shown in Table I. The size of the nanoparticles resulting from formulations containing PLGA 50:50 were consistent across different concentrations of aspirin; nanoparticle sizes averaged $\sim 113 \mathrm{~nm}$ across all three formulations containing 20,40 and 60\% aspirin. The size distribution was also consistent across the different samples indicating even-sized nanoparticles in each of the 
(a)

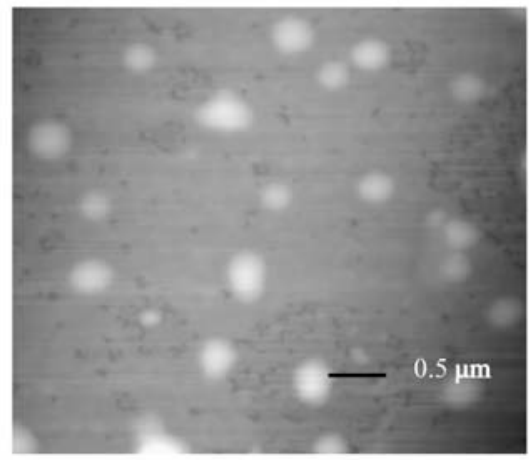

(b)

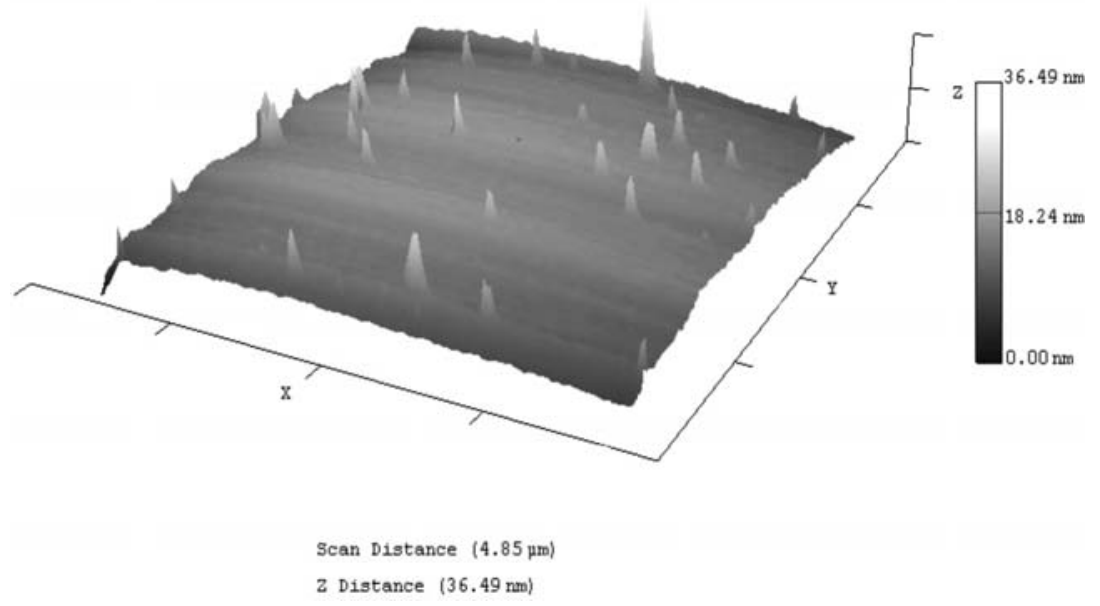

Figure 3. Atomic force microscopy (AFM) images depicting (a) surface morphology and (b) 3-D orientation of aspirin-loaded polylactide-co-glycolide (PLGA 50:50) nanoparticles.

samples tested. Similarly, formulations containing PLGA 85:15 demonstrated a mean size ranging from 113-123 nm across different formulations. However, the size distribution patterns from PLGA 85:15 formulations containing increasing amounts of aspirin (40 and 60\%) showed a significant decrease $(\sim 40 \%)$ indicating that these formulations were not evenly sized. The increasingly hydrophobic nature of PLGA 85:15 may force the nanoparticles to agglomerate resulting in a wide distribution of nanoparticles of different sizes. Overall, we determined the ideal choice of polymers from these experiments to be PLGA 50:50 due to the maintenance of particle size within a narrow range and even size distribution.

Incorporation of increasing amounts of folic acid within PLGA 50:50 showed similar results with particle sizes 123, $114,113 \mathrm{~nm}$ for 20,40 and $60 \%$ folic acid formulations, respectively as shown in Table II. The size distribution ranged from $88-93 \%$ thus indicating an even-sized particle distribution similar to aspirin nanoparticles.

Encapsulation efficiency (\%). Results from encapsulation efficiency studies demonstrated a high encapsulation of
Table I. Particle size and size distribution of the aspirinloaded PLGA polymers.

\begin{tabular}{llcc}
\hline $\begin{array}{l}\text { Amount of } \\
\text { aspirin }(\% \mathrm{w} / \mathrm{w})\end{array}$ & $\begin{array}{c}\text { Mean diameter } \\
(\mathrm{nm})\end{array}$ & $\begin{array}{c}\text { Size distribution } \\
(\%)\end{array}$ \\
\hline PLGA 50:50 & 20 & $111 \pm 23$ & 95.10 \\
& 40 & $113 \pm 16$ & 94.10 \\
& 60 & $117 \pm 25$ & 99.10 \\
PLGA 85:15 & 20 & $123 \pm 12$ & 98.40 \\
& 40 & $114 \pm 8$ & 63.80 \\
& 60 & $113 \pm 23$ & 55.40 \\
\hline
\end{tabular}

aspirin and folic acid within the polymer nanoparticles (Tables III and IV). Encapsulation efficiency was determined based upon the calculation of un-encapsulated drug present in the supernatant after preparation of nanoparticles (18). The encapsulation efficiencies for formulations prepared with increasing concentrations of drugs (aspirin and folic acid) 
Table II. Particle size and size distribution of folic acidloaded PLGA 50:50 nanoparticles.

\begin{tabular}{lcc}
\hline $\begin{array}{l}\text { Amount of folic } \\
\text { acid }(\% \mathrm{w} / \mathrm{w})\end{array}$ & $\begin{array}{c}\text { Mean diameter } \\
(\mathrm{nm})\end{array}$ & $\begin{array}{c}\text { Size distribution } \\
(\%)\end{array}$ \\
\hline 20 & $123 \pm 12$ & 88.10 \\
40 & $114 \pm 08$ & 95.20 \\
60 & $113 \pm 23$ & 92.90 \\
\hline
\end{tabular}

Table III. Encapsulation efficiency of aspirin-loaded PLGA 50:50 nanoparticles.

\begin{tabular}{lc}
\hline $\begin{array}{l}\text { Theoretical amount } \\
\text { of aspirin }(\% \mathrm{w} / \mathrm{w})\end{array}$ & $\begin{array}{l}\text { Encapsulation } \\
\text { efficiency }(\%)\end{array}$ \\
\hline 20 & 84.1 \\
40 & 80.7 \\
60 & 83.0 \\
\hline
\end{tabular}

Table IV. Encapsulation efficiency $(\%)$ of folic acid-loaded PLGA 50:50 nanoparticles.

\begin{tabular}{lc}
\hline $\begin{array}{l}\text { Theoretical amount of } \\
\text { folic acid }(\% \mathrm{w} / \mathrm{w})\end{array}$ & $\begin{array}{l}\text { Encapsulation } \\
\text { efficiency }(\%)\end{array}$ \\
\hline 20 & 82 \\
40 & 87 \\
60 & 91 \\
\hline
\end{tabular}

were within the same range $(\sim 80 \%)$. Thus, both formulations of nanoparticles demonstrated a high range of encapsulation of drugs within the polymer shells.

In vitro drug release kinetics from PLGA 50:50 nanoparticles. Figs. 4 and 5 illustrate the in vitro release profile of aspirin and folic acid, respectively, loaded on PLGA nanoparticles, in $\mathrm{pH} 7.4$ phosphate buffer. To study the effect of increasing amounts of PLGA 50:50 on drug release, various samples of PLGA 50:50 nanoparticles were studied using 1:1, 2:1 and $4: 1$ concentration of polymer to drug, respectively, for a period of 96 h (Fig. 4). A burst effect was observed in all formulations in which approximately $10 \%$ of surface drug was dissolved within the first $60 \mathrm{~min}$. The initial burst effect can be attributed to the release of the drug encapsulated near the nanosphere surface and is clearly related to the drug loading in the nanospheres. Thereafter, drug release was stable with only another $50 \%$ being released over the next 4 days, thus demonstrating a controlled release pattern, as expected. However, for formulations containing higher concentrations of PLGA (4:1), the release of the drug was further slowed. Also, with aspirin-loaded nanoparticles in 1:1 ratio, the burst release effect was higher when compared to formulations having higher polymer concentration. Similar
PLGA : Aspirin

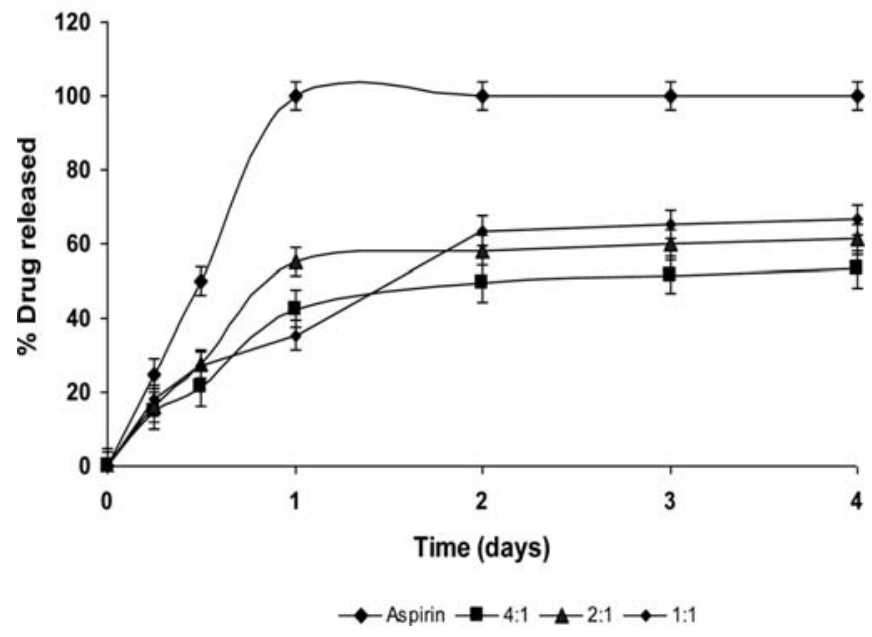

Figure 4. In vitro release of aspirin from various amounts of polylactice-coglycolide (PLGA 50:50) over a 4-day period.

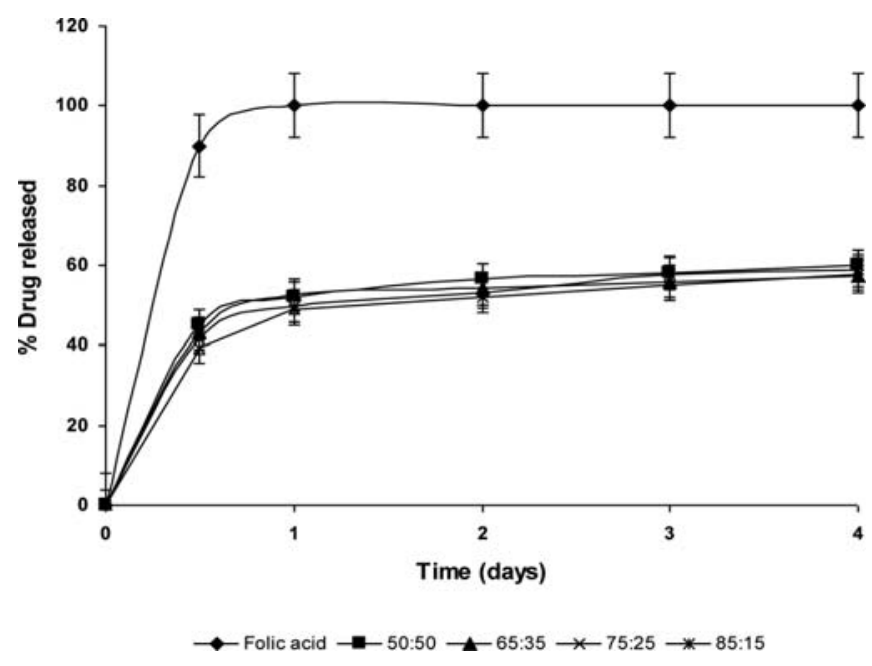

Figure 5. In vitro release of folic acid from various grades of polylactide-coglycolide (PLGA 50:50; 65:35; 75:25 and 85:15) nanoparticles over a 4-day period.

results were reported by Okada et al (22) from studies in PLGA microspheres encapsulated drugs. Overall, the controlled release of aspirin demonstrates the capability of having a sustained chemopreventive effect in the colon to prevent colon cancer.

Samples of PLGA nanoparticles containing folic acid in 1:1 concentration were prepared with various grades of PLGA (50:50; 65:35; 75:25 and 85:15). As shown in Fig. 5, drug release was prolonged from nanoparticles in relation to the free drug, however for the time period of the study there were no significant differences in release patterns from the various grades of PLGA polymers tested. While $90 \%$ of the free drug was released within $12 \mathrm{~h}$, only $45 \%$ of encapsulated drug was released using PLGA polymers over $96 \mathrm{~h}$. A burst release of folic acid was observed with all formulations. When particles were prepared by w/o/w emulsion method, 


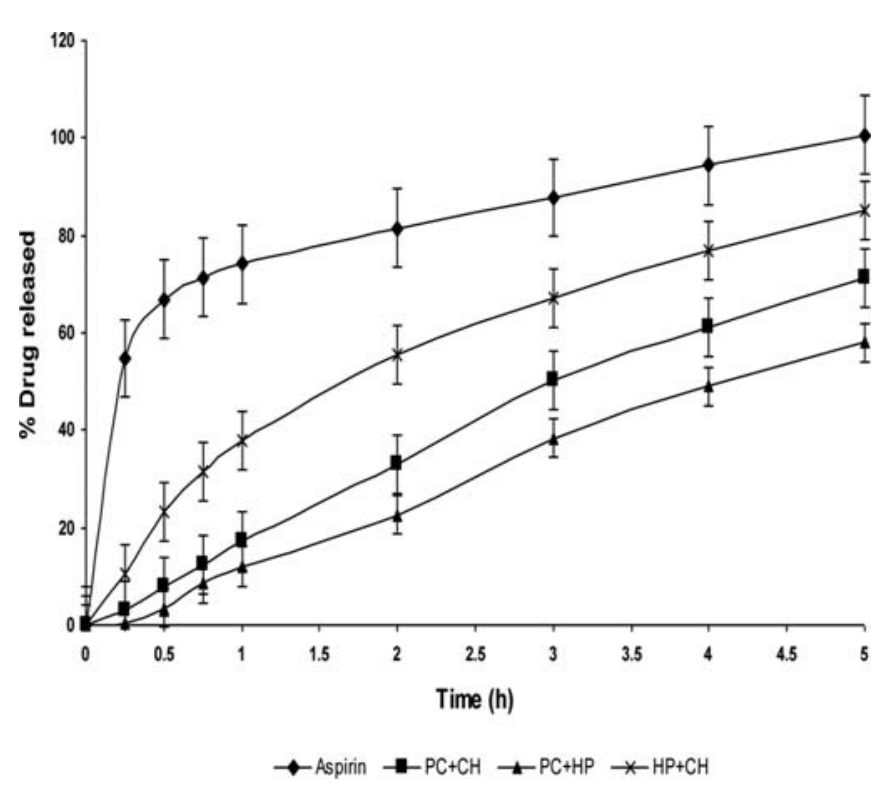

Figure 6. In vitro drug release profiles of aspirin (Asp) from combinations of conventional polymers [pectin (PC); hydroxypropyl methylcellulose (HPMC); chitosan $(\mathrm{CH})]$ in a 1:2 ratio (drug:polymer).

water soluble drugs exhibit a tendency to migrate to the aqueous medium, thereby concentrating at the surface of the particles resulting in the burst release (23). Also the burst release can be explained by the incomplete encapsulation of the drug inside nanoparticles, resulting from the unstable nature of the emulsion droplets during the solvent removal step. This potential instability may cause a part of the loaded drug to relocate at the nanoparticles surface, thereby resulting in a rapid release (24). The results showed $<10 \%$ of the drug released for the next three days.

In vitro drug release from conventional rate controlling polymers. Aspirin release from combinations of conventional polymers is shown in Fig. 6. Aspirin was combined with mixtures of polymers in 1:2 ratios. A stable release profile was observed with chitosan in combination with pectin and HPMC exhibiting higher drug release at 65 and $76 \%$, respectively, compared to pectin and HPMC combination of polymers. The slowest release of aspirin was exhibited by combinations of pectin and HPMC with approximately 55\% drug release after $5 \mathrm{~h}$. The reason for this occurrence was attributed to the presence of HPMC which has previously demonstrated a high capacity for swelling in aqueous surroundings thus slowing the release of drug from its matrix (25). Similarly, formulations of folic acid mixed in combinations of polymers (Fig. 7) demonstrated similar profiles as that of aspirin. Again, mixtures of HPMC and pectin showed the slowest release compared to other mixtures.

\section{Discussion}

Chemoprevention, using non-toxic natural or synthetic substances to decrease the risk of developing cancer, has become an important approach to decrease cancer morbidity and mortality (8). Aspirin acts by inhibiting cyclooxygenase, one of the key enzymes that convert arachidonic acid to

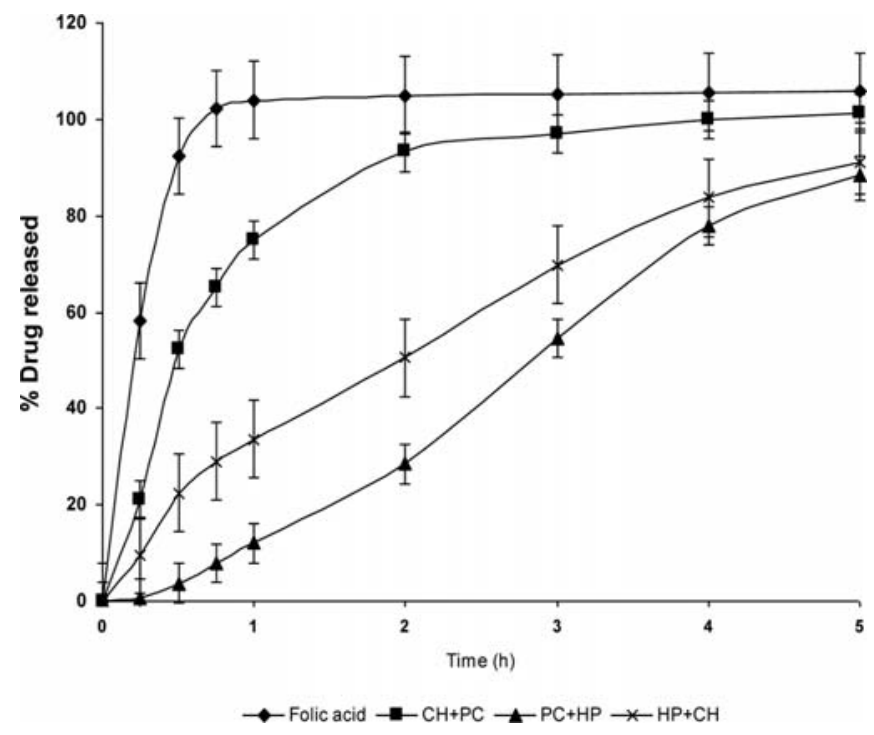

Figure 7. In vitro drug release profiles of folic acid (FA) from combinations of conventional polymers [pectin (PC); hydroxypropyl methylcellulose (HPMC); chitosan $(\mathrm{CH})$ ] in a 1:2 ratio (drug:polymer).

prostaglandins, known to play a role in cancer cell growth and development (26). The Cox-2 enzyme is absent in normal mucosa but is over-expressed in colonic, gastric and esophageal cancers as well as their precursor lesions. Thus, the inhibition of Cox-2 enzyme expression by chemopreventive agents results in suppression of colonic polyp formation. For HT-29 cell lines, this mechanism of action becomes especially significant since these cells being Cox- 2 enzyme expressive are more likely to be influenced by aspirin than other chemopreventive agents such as calcium and folic acid. Aspirin by itself demonstrated a decrease in cell viability by $\sim 20 \%$ thus confirming the mechanism of action as stated above. However, the synergistic responses were more evident when mixed with calcium and folic acid. The mechanism by which calcium exerts its chemoprevention effect is speculated to be via binding secondary bile acids in the bowel lumen rendering them insoluble; bile acids stimulate protein kinase $\mathrm{C}$ and induce cell proliferation and ornithine decarboxylase which acts as promoters in colon carcinogenesis. From our studies, it was demonstrated that calcium in combination with aspirin is potentially more effective than when used alone. Since HT-29 cell lines are Cox-2 enzyme expressive, the mechanism action of aspirin is consistent, in essence shutting down the Cox-2 enzyme system thus reducing cell viability significantly. Additional studies with a fixed concentration of aspirin $(5 \mathrm{mM})$ and combinations of folic acid at 0.5 and $1.0 \mathrm{mM}$ concentration also showed a reduction in cell viability (data not shown), however, aspirin combined with folic acid at $1.5 \mathrm{mM}$ concentration was most effective. Folic acid, a water-soluble vitamin of the B family, is essential for DNA synthesis and DNA methylation. The mechanism of action of folic acid in chemoprevention is focused largely on DNA methylation (27). Methionine is essential for maintaining the methylation status of normal cells which is crucial for transcription initiation. Depleted levels of folate can lead to hypomethylation as a result of low methionine production resulting in inappropriate activation of proto-oncogenes and 
chromosomal instability eventually leading to colon cancer. Thus, maintaining adequate levels of folic acid ensures protection of genes from mutation leading to colon cancer.

In both cell lines tested, aspirin in combination with calcium proved to have a pronounced effect in comparison with aspirin combined with folic acid, although this could be attributed to the increased concentration of calcium as compared to folic acid. Overall, the significant impact on the viability of colon cancer cells suggests that both treatment combinations are ideal for demonstration of chemopreventive effects on colon cancer.

In terms of the drug delivery aspects of this project, using drug load PLGA nanoparticles proved to be feasible for further exploration as a carrier system targeted to the colon. Poly(lactide-co-glycolide) (PLGA), an FDA approved, biodegradable co-polymer, has been widely used as matrix material for the preparation of drug-loaded nanoparticles (14). The release of drug from nanoparticles depends on the initial diffusion of the drug followed by erosion and swelling of the matrix. Subsequently, polymer degradation occurs due to hydrolysis, which is governed by the nature of copolymer composition and its molecular weight (28). Of all available grades of PLGA polymers, PLGA 50:50 co-polymer is known to hydrolyze at a faster rate than those containing a higher proportion of polylactic acid such as PLGA 75:25, thus releasing the encapsulated drug at a faster rate than other PLGA co-polymers. In vivo, these polymers undergo hydrolysis and convert into water and carbon dioxide through the citric acid cycle. Due to a relatively short duration of release study (96 h), there was incomplete release of the drug from each formulation. Hence we conclude that the release of the drug from these formulations was due to diffusion, since the degradation of the PLGA polymer to release drug takes much longer ( $>15$ days). Comparison of in vitro release performances of novel nanoparticulate PLGA formulations with that of conventional formulations containing HPMC, chitosan and pectin demonstrated that the choice of nanoparticulate drug delivery system is more feasible since they tend to release drug over longer periods of time and will probably be retained in the colonic region for longer periods of time due to its ability to adhere to the epithelial cell wall lining of the colonic lumen (29).

Strong evidence exists that chemopreventive agents are capable of preventing colon cancer, as is evidenced by the extensive literature available. Using a multi-disciplinary approach, this work investigated the synergistic effects of combinations of chemopreventive agents, namely, aspirin, calcium and folic acid, using colon cancer cell lines. In addition, in vitro studies related to the development of targeted formulations of chemopreventive combinations to the colon were carried out demonstrating the feasibility of delivering the drug to the colon region. From the preliminary results presented, we demonstrated for the first time that low concentrations of aspirin combined with either calcium or folic acid shows a synergistic effect in reduction of cell viability demonstrating the potential of this combinatorial design regimen in cancer prevention research. Future studies will assess the efficacy of triple combinations of aspirin, folic and calcium on colon cancer cell lines. A greater synergistic effect is expected which will allow the design of lower dose regimens for the targeted chemoprevention of colon cancer. In conclusion, the results obtained from both cell culture and novel formulation studies clearly demonstrate the scope of developing the chemopreventive agent combinations encapsulated in novel formulations to prevent colon cancer.

\section{Acknowledgements}

This work was partially supported by a grant from the National Institutes of Health (NIH), R03 grant ID no. 5R03CA121409 awarded to S.P. The authors also thank Dr Phyllis Nelson, California Polytechnic University, Pomona, CA for providing the AFM images.

\section{References}

1. Janne PA and Mayer RJ: Chemoprevention of colorectal cancer. New Eng J Med 342: 1960-1968, 2000.

2. Gill S and Sinicrope FA: Colorectal cancer prevention: Is an ounce of prevention worth a pound of cure? Semin Oncol 32: 24-34, 2005.

3. Serrano D, Lazzeroni M and Decensi A: Chemoprevention of colorectal cancer: an update. Tech Coloproctol 8: S248-S252, 2004.

4. Viner JL, Umar A and Hawk ET: Chemoprevention of colorectal cancer: problems, progress and prospects. Gastroenterol Clin North Am 31: 971-999, 2002.

5. Orner GA, Dashwood WM and Dashwood RH: Tumorsuppressing effects of antioxidants from tea. J Nutr 134: S3177-S3178, 2004.

6. Giovannucci E, Stampfer MJ and Colditz GA: Multi-vitamin use, folate, and colon cancer in women in the Nurses' Health Study. Ann Intern Med 129: 517-524, 1998.

7. Steinbach G, Lupton J, Reddy BS, Kral JG and Holt PR: Effect of calcium supplementation on rectal epithelial hyperproliferation in intestinal bypass subjects. Gastroenterology 106: 1162-1167, 1994.

8. Rao CV and Reddy BS: NSAIDs and chemoprevention. Curr Cancer Drug Targets 4: 29-42, 2004.

9. Borthwick GM, Johnson AS, Partington M, Burn J, Wilson R and Arthur HM: Therapeutic levels of aspirin and salicylate directly inhibit a model of angiogenesis through a Coxindependent mechanism. FASEB J 20: 2009-2016, 2006.

10. Chaudhary A, Wang J and Prabhu S: Development and validation of a high performance liquid chromatography method for the simultaneous determination of aspirin and folic acid from nano-particulate systems. Biomed Chromatogr (In press).

11. Prabhu S, Tran LP and Betageri GV: Effect of novel combinations of a co-solvent system on the controlled release of calcitonin from in situ biodegradable implants. Drug Deliv 12: 393-398, 2005.

12. Orive G, Hernandez RM, Gascon AR and Pedraz JL: Micro and nano drug delivery systems in cancer therapy. Cancer Ther 3: $131-138,2005$.

13. Otsuka H, Nagasaki Y and Kataoka K: PEGylated nanoparticles for biological and pharmaceutical applications. Adv Drug Deliv Rev 55: 403-419, 2003.

14. Panyam J and Labhasetwar V: Biodegradable nanoparticles for drug and gene delivery to cells and tissue. Adv Drug Deliv Rev 55: 329-347, 2003.

15. Cole ET, Scott RA, Connor AL, Wilding IR, Petereit HU, Schminke C, Beckert T and Cade D: Enteric coated HPMC capsules designed to achieve intestinal targeting. Int J Pharm 23: 83-95, 2002.

16. Lamprecht A, Koenig P, Ubrich N, Maincent P and Neumann D: Low molecular weight heparin nanoparticles: mucoadhesion and behaviour in Caco-2 cells. Nanotechnology 17: 3673-3680, 2006.

17. Florence AT, Hillerey AM, Hussain N and Jani PU: Nanoparticles as carriers for oral peptide absorption: studies on particle uptake and fate. J Control Release 36: 39-46, 1995.

18. Varshosaz J, Dehkordi AJ and Golafshan S: Colon-specific delivery of mesalazine chitosan microspheres. J Microencapsul 23: 329-339, 2006. 
19. Lee GM, Han BK, Kim JH and Palsson BO: Effect of calcium chloride treatment on hybridoma cell viability and growth. Biotechnol Lett 14: 891-896, 1992.

20. Yu HG, Huang JA and Yang YN: The effects of acetylsalicylic acid on proliferation, apoptosis, and invasion of cylcooxygenase-2 negative colon cancer cells. Eur J Clin Invest 32: 838-846, 2002.

21. Mainardes RM and Evangelista RC: PLGA nanoparticles containing praziquantel: effect of formulation variables on size distribution. Int J Pharm 290: 137-144, 2005.

22. Okada H: One- and three-month release injectable microspheres of the LH-RH superagonist leuprorelin acetate. Adv Drug Deliv Rev 28: 43-70, 1997.

23. Hans ML and Lowman AM: Biodegradable nanoparticles for drug delivery and targeting. Curr Opin Solid State Mater Sci 6: 319-327, 2002.

24. Lu W and Park TG: Protein release from poly(lactic-co-glycolic acid) microspheres: protein stability problems. PDA J Pharm Sci Technol 49:13-19, 1995.
25. Vlachou M, Naseef $H$ and Efentakis $M$ : Swelling properties of various polymers used in controlled release systems. J Biomater Appl 16: 125-138, 2001

26. Sturmer T, Glynn RJ, Manson JE, Buring JE and Hennekens CH: Aspirin use and colorectal cancer: Post-trial follow-up data from the Physicians' Health Study. Ann Intern Med 128: 713-720, 1998.

27. Lamprecht A and Lipkin M: Chemoprevention of colon cancer by calcium, vitamin $\mathrm{D}$ and folate: molecular mechanisms. Nat Rev Cancer 3: 601-614, 2004

28. Dong Y and Feng SS: Nanoparticles of poly(d,l-lactide)/ methoxy poly(ethyleneglycol)-poly(d,l-lactide) blends for controlled release of paclitaxel. J Biomed Mater Res 78: 12-19, 2006.

29. Lamprecht A, Schafer U and Lehr CM: Size-dependent bioadhesion of micro- and nanoparticulate carriers to the inflamed colonic mucosa. Pharm Res 18: 788-793, 2001. 